\title{
Kingman 溯祖过程的积分表示
}

\author{
周友洲 \\ 西交利物浦大学数学科学系, 苏州 215123 \\ E-mail: youzhou.zhou@xjtlu.edu.cn
}

收稿日期: 2019-09-19; 接受日期: 2019-12-19; 网络出版日期: 2020-07-15

国家自然科学基金 (批准号: 11701570) 资助项目

\begin{abstract}
摘要 Kingman 溯祖过程是种群遗传学中的首个溯祖模型. 目前, 它已经成为一个基准溯祖模型, 对 它的研究也相当深入. 本文的主要目的是得到其概率分布的一个复积分表示. 然后, 利用该积分表示 得到小时间局部中心极限定理的任意阶渐近展开式.
\end{abstract}

\section{关键词 Kingman 溯祖过程 积分表示 局部中心极限定理 \\ MSC (2020) 主题分类 $60 \mathrm{~F} 10,60 \mathrm{C} 05$}

\section{1 引言}

Kingman 溯祖过程是种群遗传学中的首个溯祖模型. Kingman ${ }^{[1]}$ 首次提出该模型, 并用它来描述 样本的家谱结构. 随后该模型被广泛应用于生物学. 后来, $\Lambda$ 溯祖过程 ${ }^{[2]}$ 等推广的溯祖模型也被陆续 提出. 关于溯祖过程的研究进展, 可参见文献 [3].

一般而言, 种群遗传学模型有两类: 要么是描述种群随时间向前推移的发展情况, 要么是沿时间 往回追溯的发展情况. 这两类模型存在一种对偶关系. 事实上, 这类溯祖结构在自然界非常普遍, 文 献 [4] 是一个很好的综述文章, 介绍了物理学和化学中溯祖结构的数学模型. 另外, 溯祖模型也有不同 的诠释方式, 例如, Evans ${ }^{[5]}$ 将 Kingman 溯祖过程作为随机度量空间进行了研究.

本文将 Kingman 溯祖过程作为一个连续时间 Markov 链来研究. 当沿时间往回看时, 它描述了种 群的家谱演化规律. 个体间的姊妹关系就是一种等价关系, 因此, 可以按照姊妹关系对样本进行分类. Kingman 溯祖过程刻画了这些分类的演化规律. 为此, 记 $[n]=\{1,2, \ldots, n\}$ 为一样本量为 $n$ 的样本. 按照姊妹关系, 可以得到该样本的一个分划. 记 $A_{n}$ 为该样本所有可能的分划全体. Kingman 溯祖过 程就是取值于 $A_{n}$ 的连续时间 Markov 链. 如果只关心样本中的家庭数, 那么就得到一个取整数值的 
纯死 Markov 链, 记为 $D_{t}^{n}$. 该 Markov 链的转移速率为

$$
q_{n, m}= \begin{cases}\frac{n(n+\theta-1)}{2}, & m=n-1, \\ -\frac{n(n+\theta-1)}{2}, & m=n, \\ 0, & m \neq n, n-1,\end{cases}
$$

这里 $\theta>0$ 是变异率. 当 $n \rightarrow \infty$ 时, Markov 链 $D_{t}^{n}$ 会收玫于一个带流入边界 $\infty$ 的纯死 Markov 链. 它 的有限时间分布是 $d_{n}^{\theta}(t)=\mathrm{P}\left(D_{t}=n\right)$. 有趣的是, 这些概率出现在一类特殊 Fleming-Viot 过程的转移 函数中 (参见文献 [6]). 它们刻画了原始祖先的基因在进化过程中是如何丢失的. 在 1984 年, Tavaré ${ }^{[7]}$ 得到了 $d_{n}^{\theta}(t)$ 的级数表达式

$$
\begin{aligned}
& d_{0}^{\theta}(t)=1-\sum_{k=1}^{\infty} \frac{2 k-1+\theta}{k !}(-1)^{k-1} \theta_{(k-1)} \mathrm{e}^{-\lambda_{k} t}, \\
& d_{n}^{\theta}(t)=\sum_{k=1}^{\infty} \frac{2 k-1+\theta}{k !}(-1)^{k-n}\left(\begin{array}{c}
k \\
n
\end{array}\right)(n+\theta)_{(k-1)} \mathrm{e}^{-\lambda_{k} t}, \quad n \geqslant 1, \\
& \lambda_{k}=\frac{k(k+\theta-1)}{2}, \quad \theta_{(k)}=\frac{\Gamma(\theta+k)}{\Gamma(\theta)}, \quad k \geqslant 0 .
\end{aligned}
$$

30 多年过去了, 很少有人利用该级数表达式来进行分析, 只是用该级数表达式的估计得到了一类特殊 Fleming-Viot 过程及其原子过程的遍历不等式 (参见文献 [8]).

Kingman 溯祖过程的突出特点就是它具有流入边界 $\infty$. 该过程会瞬间从状态 $\infty$ 转移到有限状 态. 很多的细节被隐藏在转移过程中, 因此, 我们有必要研究它在时间靠近 0 时的行为. 事实上, 已经 有很多关于小时间行为的研究, 如文献 [9-12]. 然而, 很少有人利用 $d_{n}^{\theta}(t)$ 的级数表达式. 因为那些级 数表达式都是交错级数, 当时间 $t$ 靠近 0 时, 每一项都不可忽略, 所以, 无法利用截断的技巧来进行渐 近行为的研究. 已有的研究结果都是通过鞅论等概率工具来进行研究. 但是, 要得到更加精细的渐近分 析结果, 最好直接利用 $d_{n}^{\theta}(t)$ 的显式表达式进行计算.

为此, 本文得到 $d_{n}^{\theta}(t)$ 的复积分表达式. 通过复分析中的渐近分析手段, 可以得到 $d_{n}^{\theta}(t)$ 的一些 精细的渐近分析结果. 为了得到复积分表达式, 我们的基本想法是将级数表达式中的一些组合系数及 $\mathrm{e}^{-\lambda_{k} t}$ 用复积分代替. 因为组合系数可以用 Cauchy 积分公式来表示, 而 $\mathrm{e}^{-\lambda_{k} t}$ 可以用 Fourier 变换来 表示. 替换之后, 级数变成了积分, 而积分的被积函数就变成了几何级数. 对几何级数求和后, 我们就 得到一个容易处理的复积分. 处理复积分的一般方法就是最速下降法, 但是, 在应用最速下降法之前, 需要对积分进行一些围道变形等预处理. 本文得到一个局部中心极限定理. Limic 和 Talarczyk ${ }^{[11,12]}$ 得到了小时间泛函中心极限定理. 但是, 局部中心极限定理无法从泛函中心极限定理得到, 更不用提 其无穷阶渐进展开式. Depperschmidt 等 ${ }^{[9]}$ 讨论了 $D_{t}$ 的小时间大偏差原理. 我们相信利用复积分表 示也可以得到小时间大偏差及中偏差原理.

本文将先讨论复积分表示及其证明. 需要指出的是, 本文的复积分表示同文献 [13] 中的复积分 (2.16) 本质上是相同的, 只是证明方式不一样. 另外, 本文在定理 3.2 也给出了另外一个等价的复积分 表示, 它更适合做渐近分析. 我们通过最速下降法得到了局部中心极限定理. 定理 3.2 中的积分表示是 全新的. 最后, 将给出相关引理的证明.

本文中, 记号 “ ” 多次出现. 定义 $a(t) \sim b(t)$ 为

$$
\lim _{t \rightarrow 0} \frac{a(t)}{b(t)}=1 \text {. }
$$


很多常数 $M_{1}$ 和 $M_{2}$ 也多次出现. 我们不关注常数间的关系, 仅将它们看成不依赖参数 $t$ 的常数. 另 外, 符号 $\mathrm{i}$ 仅用来表示复数的虚单位, 即 $\mathrm{i}^{2}=-1$.

\section{$2 d_{n}^{\theta}(t)$ 的复积分表示}

本节将探讨 $d_{n}^{\theta}(t)$ 的积分表示. 一般而言, 积分要比级数更易于处理. 因此, 如果要利用 $d_{n}^{\theta}(t)$ 的 表达式来研究 $D_{t}$ 的小时间行为, 我们倾向于将 $d_{n}^{\theta}(t)$ 的级数表达式换成积分表达式.

由于 $d_{n}^{\theta}(t)$ 的级数表达式中涉及组合系数和 $\mathrm{e}^{-\lambda_{k} t}$, 二者可以分别通过 Cauchy 积分公式和 Fourier 变换变成复积分. 这些复积分的围道要小心选取以保证级数的收玫性, 然后, 级数就变成一个复积 分, 该积分的被积函数就是一个几何级数. 随后, 我们可以通过计算几何级数得到一个完整的复积分 表示.

定理 2.1 对于任意 $n \geqslant 0, d_{n}^{\theta}(t)$ 具有如下积分表示:

$$
d_{n}^{\theta}(t)=\left(\begin{array}{c}
2 n-1+\theta \\
n
\end{array}\right) \frac{1}{2^{2 n-1+\theta}} \mathrm{e}^{\frac{(1-\theta)^{2} t}{8}} \frac{1}{\sqrt{2 \pi t}} \int_{-\infty-\mathrm{i} A}^{\infty-\mathrm{i} A} \mathrm{e}^{-\frac{w^{2}}{2 t}} \frac{\mathrm{i} \sin \frac{w}{2}}{\left(\cos \frac{w}{2}\right)^{2 n+\theta}} d w
$$

这里 $A>0$ 并且 $\left(\cos \frac{w}{2}\right)^{2 n+\theta}$ 定义为幕函数 $z^{2 n+\theta}$ 的主值分支.

证明 首先, 由简单代数运算, 有

$$
\frac{2 k-1+\theta}{k !}(-1)^{k-n}\left(\begin{array}{l}
k \\
n
\end{array}\right)(n+\theta)_{(k-1)}=(-1)^{k-n}\left[\frac{(n+\theta)_{(k)}}{k !}\left(\begin{array}{l}
k \\
n
\end{array}\right)+\frac{(n+\theta)_{(k-1)}}{(k-1) !}\left(\begin{array}{c}
k-1 \\
n
\end{array}\right)\right]
$$

和

$$
\lambda_{k}=\frac{1}{2}\left(k^{2}+(\theta-1) k\right)=\frac{1}{2}\left[k+\frac{\theta-1}{2}\right]^{2}-\frac{(\theta-1)^{2}}{8} .
$$

由 Fourier 变换可知,

$$
\mathrm{e}^{-\lambda_{k} t}=\exp \left\{\frac{(\theta-1)^{2} t}{8}\right\} \frac{1}{\sqrt{2 \pi}} \int_{-\infty-\mathrm{i} a}^{\infty-\mathrm{i} a} \exp \left\{-\frac{\xi^{2}}{2}-\mathrm{i} \xi\left(\frac{\theta-1}{2}\right) \sqrt{t}\right\} \mathrm{e}^{-\mathrm{i} \xi \sqrt{t} k} d \xi
$$

这里 $a>\frac{\log \frac{3}{2}}{\sqrt{t}}$. 另外, 由 Cauchy 积分公式易知,

$$
\begin{aligned}
& \frac{(n+\theta)_{(k)}}{k !}=\frac{1}{2 \pi \mathrm{i}} \int_{C_{1}} \frac{\xi_{1}^{n+\theta+k-1}}{\left(\xi_{1}-1\right)^{k+1}} d \xi_{1}, \\
& \left(\begin{array}{c}
k \\
n
\end{array}\right)=\frac{1}{2 \pi \mathrm{i}} \int_{C_{2}} \frac{\xi_{2}^{k}}{\left(\xi_{2}-1\right)^{n+1}} d \xi_{2},
\end{aligned}
$$

此处, $C_{1}$ 和 $C_{2}$ 均是圆心在 1 的圆周, $C_{1}$ 的半径满足 $r>\frac{3}{2 \mathrm{e}^{\sqrt{t} a}-3}, C_{2}$ 的半径为 $\frac{1}{2}$. 这些积分围道的选 取是为了保证级数的收玫性. 如果把 $d_{n}^{\theta}(t)$ 的级数表达式中的组合系数和 $\mathrm{e}^{-\lambda_{k} t}$ 用它们的积分表达式 (2.1)-(2.3) 替换掉, 则得到

$$
\begin{aligned}
d_{n}^{\theta}(t)= & \sum_{k=n}^{\infty}(-1)^{k-n} \frac{1}{(2 \pi \mathrm{i})^{2}}\left[\int_{C_{1}} \int_{C_{2}} \frac{\xi_{1}^{n+\theta+k-1}}{\left(\xi_{1}-1\right)^{k+1}} d \xi_{1} \frac{\xi_{2}^{k}}{\left(\xi_{2}-1\right)^{n+1}} d \xi_{2}\right. \\
& \left.+\int_{C_{1}} \int_{C_{2}} \frac{\xi_{1}^{n+\theta+k-2}}{\left(\xi_{1}-1\right)^{k}} d \xi_{1} \frac{\xi_{2}^{k-1}}{\left(\xi_{2}-1\right)^{n+1}} d \xi_{2}\right] \exp \left\{\frac{(\theta-1)^{2} t}{8}\right\}
\end{aligned}
$$




$$
\begin{aligned}
& \times \frac{1}{\sqrt{2 \pi}} \int_{-\infty-\mathrm{i} a}^{\infty-\mathrm{i} a} \exp \left\{-\frac{\xi_{3}^{2}}{2}-\mathrm{i} \xi_{3}\left(\frac{\theta-1}{2}\right) \sqrt{t}\right\} \mathrm{e}^{-\mathrm{i} \xi_{3} \sqrt{t} k} d \xi_{3} \\
= & \frac{1}{\sqrt{2 \pi}(2 \pi \mathrm{i})^{2}} \exp \left\{\frac{(\theta-1)^{2} t}{8}\right\} \sum_{k=n}^{\infty} \int_{C_{1}} \int_{C_{2}} \int_{-\infty-\mathrm{i} a}^{\infty-\mathrm{i} a} \exp \left\{-\frac{\xi_{3}^{2}}{2}-\mathrm{i} \xi_{3}\left(\frac{2 n+\theta-1}{2}\right) \sqrt{t}\right\} \\
& \times\left[-\frac{\xi_{1} \xi_{2}}{\mathrm{e}^{\mathrm{i} \xi_{3} \sqrt{t}}\left(\xi_{1}-1\right)}\right]^{k-n} \frac{\xi_{1}^{2 n-2+\theta} \xi_{2}^{n-1}\left(\xi_{1} \xi_{2}+\xi_{1}-1\right)}{\left(\xi_{1}-1\right)^{n+1}\left(\xi_{2}-1\right)^{n+1}} d \xi_{1} d \xi_{2} d \xi_{3} .
\end{aligned}
$$

上述围道满足条件

$$
\left|\frac{\xi_{1} \xi_{2}}{\mathrm{e}^{\mathrm{i} \xi_{3} \sqrt{t}}\left(\xi_{1}-1\right)}\right|<1
$$

因此, 级数一致收玫. 可以交换积分和求和顺序, 进而得到一个复积分, 其被积函数只是几何级数. 通 过计算几何级数, 得到

$$
\begin{aligned}
d_{n}^{\theta}(t)= & \frac{1}{\sqrt{2 \pi}(2 \pi \mathrm{i})^{2}} \exp \left\{\frac{(\theta-1)^{2} t}{8}\right\} \int_{C_{1}} \int_{C_{2}} \int_{-\infty-\mathrm{i} a}^{\infty-\mathrm{i} a} \exp \left\{-\frac{\xi_{3}^{2}}{2}-\mathrm{i} \xi_{3}\left(\frac{2 n+\theta-3}{2}\right) \sqrt{t}\right\} \\
& \times \frac{\xi_{1} \xi_{2}+\xi_{1}-1}{\xi_{1} \xi_{2}+\mathrm{e}^{\mathrm{i} \xi_{3} \sqrt{t}}\left(\xi_{1}-1\right)} \frac{\xi_{1}^{2 n-2+\theta} \xi_{2}^{n-1}}{\left(\xi_{1}-1\right)^{n}\left(\xi_{2}-1\right)^{n+1}} d \xi_{1} d \xi_{2} d \xi_{3} \\
= & \frac{1}{\sqrt{2 \pi}(2 \pi \mathrm{i})^{2}} \exp \left\{\frac{(\theta-1)^{2} t}{8}\right\} \int_{-\infty-\mathrm{i} a}^{\infty-\mathrm{i} a} \exp \left\{-\frac{\xi_{3}^{2}}{2}-\mathrm{i} \xi_{3}\left(\frac{2 n+\theta-3}{2}\right) \sqrt{t}\right\} d \xi_{3} \\
& \times \int_{C_{2}} \frac{\xi_{2}^{n-1}}{\left(\xi_{2}+\mathrm{e}^{\mathrm{i} \sqrt{t} \xi_{3}}\right)\left(\xi_{2}-1\right)^{n+1}} d \xi_{2} \int_{C_{1}} \frac{\left(\xi_{1} \xi_{2}+\xi_{1}-1\right) \xi_{1}^{2 n-2+\theta}}{\left(\xi_{1}-1\right)^{n}\left(\xi_{1}-\frac{\mathrm{e}^{\mathrm{i} \sqrt{t} \xi_{3}}}{\left.\xi_{2}+\mathrm{e}^{\mathrm{i} \sqrt{t} \xi_{3}}\right)} d \xi_{1} .\right.}
\end{aligned}
$$

下面通过留数计算化简上述积分. 首先积掉 $\xi_{1}$, 于是,

$$
\frac{1}{2 \pi \mathrm{i}} \int_{C_{1}} \frac{\left(\xi_{1} \xi_{2}+\xi_{1}-1\right) \xi_{1}^{2 n-2+\theta}}{\left(\xi_{1}-1\right)^{n}\left(\xi_{1}-\frac{\mathrm{e}^{\mathrm{i} \sqrt{t} \xi_{3}}}{\xi_{2}+\mathrm{e}^{\mathrm{i} \sqrt{t} \xi_{3}}}\right)} d \xi_{1}=R_{1}\left(\xi_{2}, \xi_{3}\right)+R_{2}\left(\xi_{2}, \xi_{3}\right),
$$

这里 $R_{1}\left(\xi_{2}, \xi_{3}\right)$ 和 $R_{2}\left(\xi_{2}, \xi_{3}\right)$ 分别是 $\xi_{1}=1$ 和 $\xi_{1}=\frac{\mathrm{e}^{\mathrm{i} \sqrt{t} \xi_{3}}}{\xi_{2}+\mathrm{e}^{\mathrm{i} \sqrt{t} \xi_{3}}}$ 处的留数, 并且

$$
\begin{aligned}
R_{1}\left(\xi_{2}, \xi_{3}\right)= & \sum_{l=0}^{n-1}(-1)^{n-l-1}(n-l-1) !\left(\begin{array}{c}
n-1 \\
l
\end{array}\right)\left[\frac{(2 n-1+\theta) !}{(2 n-l-1+\theta) !}\left(\xi_{2}+1\right)-\frac{(2 n-2+\theta) !}{(2 n-l-2+\theta) !}\right] \\
& \times\left(\frac{\xi_{2}+\mathrm{e}^{\mathrm{i} \xi_{3} \sqrt{t}}}{\xi_{2}}\right)^{n-l}, \\
R_{2}\left(\xi_{2}, \xi_{3}\right)= & (-1)^{n} \frac{\left(\mathrm{e}^{\mathrm{i} \xi_{3} \sqrt{t}}-1\right) \mathrm{e}^{\mathrm{i} \xi_{3} \sqrt{t}(2 n-2+\theta)}}{\xi_{2}^{n-1}\left(\xi_{2}+\mathrm{e}^{\mathrm{i} \xi_{3} \sqrt{t}}\right)^{n-1+\theta}} .
\end{aligned}
$$

所以,

$$
\begin{aligned}
d_{n}^{\theta}(t)= & \frac{1}{\sqrt{2 \pi}(2 \pi \mathrm{i})^{2}} \exp \left\{\frac{(\theta-1)^{2} t}{8}\right\} \int_{-\infty-\mathrm{i} a}^{\infty-\mathrm{i} a} \exp \left\{-\frac{\xi_{3}^{2}}{2}-\mathrm{i} \xi_{3}\left(\frac{2 n+\theta-3}{2}\right) \sqrt{t}\right\} d \xi_{3} \\
& \times \int_{C_{2}} \frac{\xi_{2}^{n-1}}{\left(\xi_{2}+\mathrm{e}^{\mathrm{i} \sqrt{t} \xi_{3}}\right)\left(\xi_{2}-1\right)^{n+1}}\left[R_{1}\left(\xi_{2}, \xi_{3}\right)+R_{2}\left(\xi_{2}, \xi_{3}\right)\right] d \xi_{2} .
\end{aligned}
$$

易证

$$
\frac{1}{2 \pi \mathrm{i}} \int_{C_{2}} \frac{\xi_{2}^{n-1}}{\left(\xi_{2}+\mathrm{e}^{\mathrm{i} \sqrt{t} \xi_{3}}\right)\left(\xi_{2}-1\right)^{n+1}} R_{1}\left(\xi_{2}, \xi_{3}\right) d \xi_{2}=0
$$


因为在被积函数中, 分子的最高次是 $n-1$, 而分母的最高次是 $n+1$. 另外,

$$
\begin{aligned}
& \frac{1}{2 \pi \mathrm{i}} \int_{C_{2}} \frac{\xi_{2}^{n-1}}{\left(\xi_{2}+\mathrm{e}^{\mathrm{i} \sqrt{t} \xi_{3}}\right)\left(\xi_{2}-1\right)^{n+1}} R_{2}\left(\xi_{2}, \xi_{3}\right) d \xi_{2} \\
& \quad=(-1)^{n}\left(\mathrm{e}^{\mathrm{i} \xi_{3} \sqrt{t}}-1\right) \mathrm{e}^{\mathrm{i} \xi_{3} \sqrt{t}(2 n-2+\theta)} \frac{1}{2 \pi \mathrm{i}} \int_{C_{2}} \frac{1}{\left(\xi_{2}-1\right)^{n+1}\left(\xi_{2}+\mathrm{e}^{\mathrm{i} \xi_{3} \sqrt{t}}\right)^{n+\theta}} d \xi_{2} \\
& \quad=\left(\mathrm{e}^{\mathrm{i} \xi_{3} \sqrt{t}}-1\right) \mathrm{e}^{\mathrm{i} \xi_{3} \sqrt{t}(2 n-2+\theta)}\left(\begin{array}{c}
2 n-1+\theta \\
n
\end{array}\right)\left(1+\mathrm{e}^{\mathrm{i} \xi_{3} \sqrt{t}}\right)^{-(2 n+\theta)},
\end{aligned}
$$

此处, 围道 $C_{2}$ 中只包含一个奇点 1 , 另外一个奇点 $\xi_{2}=-\mathrm{e}^{\mathrm{i} \xi_{3} \sqrt{t}}$ 在围道 $C_{2}$ 之外. 因此,

$$
\begin{aligned}
d_{n}^{\theta}(t)= & \frac{1}{\sqrt{2 \pi}} \exp \left\{\frac{(\theta-1)^{2} t}{8}\right\} \int_{-\infty-\mathrm{i} a}^{\infty-\mathrm{i} a} \exp \left\{-\frac{\xi_{3}^{2}}{2}-\mathrm{i} \xi_{3}\left(\frac{2 n+\theta-3}{2}\right) \sqrt{t}\right\} \\
& \times \mathrm{e}^{\mathrm{i} \xi_{3} \sqrt{t}(2 n-2+\theta)}\left(\mathrm{e}^{\mathrm{i} \xi_{3} \sqrt{t}}-1\right)\left(\begin{array}{c}
2 n-1+\theta \\
n
\end{array}\right)\left(1+\mathrm{e}^{\mathrm{i} \xi_{3} \sqrt{t}}\right)^{-(2 n+\theta)} d \xi_{3} \\
= & \left(\begin{array}{c}
2 n-1+\theta \\
n
\end{array}\right) \exp \left\{\frac{(\theta-1)^{2} t}{8}\right\} \frac{1}{\sqrt{2 \pi}} \int_{-\infty-\mathrm{i} a}^{\infty-\mathrm{i} a} \\
& \times \exp \left\{-\frac{\xi_{3}^{2}}{2}+\mathrm{i} \xi_{3}\left(\frac{2 n+\theta-1}{2}\right) \sqrt{t}\right\} \frac{\left(\mathrm{e}^{\mathrm{i} \xi_{3} \sqrt{t}}-1\right)}{\left(1+\mathrm{e}^{\mathrm{i} \xi_{3} \sqrt{t}}\right)^{(2 n+\theta)}} d \xi_{3} .
\end{aligned}
$$

考虑变量代换 $w=\xi_{3} \sqrt{t}$, 有

$$
d_{n}^{\theta}(t)=\left(\begin{array}{c}
2 n-1+\theta \\
n
\end{array}\right) \frac{1}{2^{2 n-1+\theta}} \mathrm{e}^{\frac{(1-\theta)^{2} t}{8}} \frac{1}{\sqrt{2 \pi t}} \int_{-\infty-\mathrm{i} A}^{\infty-\mathrm{i} A} \mathrm{e}^{-\frac{w^{2}}{2 t}} \frac{\mathrm{i} \sin \frac{w}{2}}{\left(\cos \frac{w}{2}\right)^{2 n+\theta}} d w,
$$

这里 $A=a \sqrt{t}>\log (3 / 2)$. 事实上, 关于 $A$ 的限制可以去掉, 因为对于 $w=R+\mathrm{i} y, y \in\left[A^{\prime}, A\right]$,

$$
\left|\mathrm{e}^{-\frac{w^{2}}{2 t}} \frac{\mathrm{i} \sin \frac{w}{2}}{\left(\cos \frac{w}{2}\right)^{2 n+\theta}}\right| \leqslant M \mathrm{e}^{-\frac{R^{2}}{2 t}} .
$$

证毕.

\section{3 局部中心极限定理}

由文献 [3] 可知 $\lim _{t \rightarrow 0} t D_{t}=2$. 当 $\theta=0$ 时, Limic 和 Talarczyk [11,12] 得到了小时间泛函中心极 限定理, 即当 $\epsilon \rightarrow 0$ 时, $\epsilon^{-\frac{1}{2}}\left(\frac{\epsilon t}{2} D_{\epsilon t}-1\right)$ 弱收玫于 Gauss 过程 $\frac{1}{\sqrt{2} t} \int_{0}^{t} s d B_{s}, t \geqslant 0$. 自然地, $D_{t}$ 的中心 极限定理是成立的, 即 $\frac{\left(t D_{t}-2\right)}{\sqrt{t}}$ 弱收敛于正态分布. 所谓局部中心极限定理就是考虑 $\mathrm{P}\left(D_{t}=\left[\frac{2+\sqrt{t} x}{t}\right]\right)$ 在 $t \rightarrow 0$ 时可以用正态分布密度函数来逼近. 本节将得到 $\mathrm{P}\left(D_{t}=\left[\frac{2+\sqrt{t} x}{t}\right]\right)$ 的渐近展开式. 通常地, $\mathrm{P}\left(D_{t}=\left[\frac{2+\sqrt{t} x}{t}\right]\right)$ 的渐近展开式是指 $\sum_{k=0}^{\infty} \varphi_{k}(t)$, 这里 $\varphi_{k+1}(t)=o\left(\varphi_{k}(t)\right)$, 并且对于任意正整数 $m$, 存 在常数 $M>0$ 和 $\delta>0$, 使得

$$
\left|\mathrm{P}\left(D_{t}=\left[\frac{2+\sqrt{t} x}{t}\right]\right)-\sum_{k=0}^{m} \varphi_{k}(t)\right| \leqslant M \varphi_{k+1}(t), \quad \forall 0<t<\delta .
$$

通常将上述展开式简记为

$$
\mathrm{P}\left(D_{t}=\left[\frac{2+\sqrt{t} x}{t}\right]\right) \sim \sum_{k=0}^{\infty} \varphi_{k}(t)
$$


定理 3.1 对于正整数 $n=\left[\frac{2+x \sqrt{t}}{t}\right], x \in \mathbb{R}$, 令 $\sigma^{2}=\frac{(2 n+\theta) t}{6}, v=\frac{(2 n+\theta) t-4}{2 \sqrt{t}}$, 有

$$
\mathrm{P}\left(D_{t}=n\right) \sim \sum_{k=0}^{\infty}(-1)^{k}\left[d_{k}(v) \frac{1}{\sqrt{2 \pi} \sigma} \mathrm{e}^{-\frac{v^{2}}{2 \sigma^{2}}}\right] t^{(k+1) / 2},
$$

这里

$$
\begin{aligned}
& d_{k}(v)=\mathrm{e}^{\frac{(1-\theta)^{2} t}{8}}\left(\begin{array}{c}
2 n-1+\theta \\
n
\end{array}\right) \frac{\sqrt{2 \pi}}{\sqrt{t} \sigma^{k} 2^{2 n-1+\theta}} \sum_{l=0}^{k} \frac{1}{(2(k-l)+1) !}\left\{\sum_{\mathcal{S}_{l}} \frac{\left[-6 a_{i} / i !\right]^{m_{i}}}{m_{i} !} H_{l+2 \sum_{i=1}^{l} m_{i}}\left(\frac{v}{\sigma}\right)\right\} \\
& a_{k}=\frac{2^{2 k+4}\left(2^{2 k+4}-1\right) B_{2 k+4} k !}{(2 k+4)(2 k+4) !} \\
& \mathcal{S}_{l}=\left\{\left(m_{1}, \ldots, m_{l}\right) \in \mathbb{N}^{l} \mid \sum_{i=1}^{l} i m_{i}=l\right\},
\end{aligned}
$$

并且 $H_{l}(x)=(-1)^{l} \mathrm{e}^{\frac{x^{2}}{2}} \frac{d^{l}}{d x^{l}}\left[\mathrm{e}^{-\frac{x^{2}}{2}}\right]$ 是 Hermite 多项式, $B_{2 k}$ 是 Bernoulli 数.

定理 3.1 的证明主要是利用最速下降法. 但是, 定理 2.1 中的积分表达式不适合做渐近分析, 需要 通过围道变形得到一个等价的易于处理的积分表示.

定理 3.2 对于 $n \geqslant 0, d_{n}^{\theta}(t)$ 有如下积分表示:

$$
d_{n}^{\theta}(t)=\mathrm{P}\left(D_{t}=n\right)=\left(\begin{array}{c}
2 n-1+\theta \\
n
\end{array}\right) \frac{c_{n}^{\theta}(t)}{2^{2 n-1+\theta}},
$$

这里

$$
\begin{aligned}
c_{n}^{\theta}(t) & =\mathrm{e}^{\frac{(1-\theta)^{2} t}{8}} \frac{\mathrm{i}}{2 \sqrt{2 \pi t}} \int_{C}\left[K_{t}(z) \phi_{t}(z)-K_{t}(-z) \phi_{t}(-z)\right] d z, \\
K_{t}(z) & =\mathrm{e}^{-\frac{(\pi+z)^{2}}{2 t}} \frac{\sin \left(\frac{z+\pi}{2}\right)}{\cos ^{2 n+\theta}\left(\frac{z+\pi}{2}\right)}, \\
\phi_{t}(z) & =1+2 \sum_{k=1}^{\infty} \mathrm{e}^{-\frac{8 \pi^{2} k^{2}}{t}} \cosh \frac{4 k \pi(\pi+z)}{t},
\end{aligned}
$$

$C$ 是复平面上的单位圆周.

证明 考虑函数 $f(w)=\mathrm{e}^{-\frac{w^{2}}{2 t}} \frac{\mathrm{i} \sin \frac{w}{2}}{\left(\cos \frac{w}{2}\right)^{2 n+\theta}}$. 易证 $f(-w)=-f(w)$. 因此,

$$
\int_{-\infty-\mathrm{i} A}^{\infty-\mathrm{i} A} f(w) d w=\int_{\infty+\mathrm{i} A}^{-\infty+\mathrm{i} A}-f(-w) d w=\int_{\infty+\mathrm{i} A}^{-\infty+\mathrm{i} A} f(w) d w .
$$

那么,

$$
2 \int_{-\infty-\mathrm{i} A}^{\infty-\mathrm{i} A} f(w) d w=\int_{-\infty-\mathrm{i} A}^{\infty-\mathrm{i} A} f(w) d w+\int_{\infty+\mathrm{i} A}^{-\infty+\mathrm{i} A} f(w) d w=\int_{\mathcal{L}} f(w) d w
$$

这里的围道 $\mathcal{L}$ 是两根水平的平行线. 于是, 可以将 $\mathcal{L}$ 往水平坐标轴移动, 最后变成图 1 中的围道.

虽然区间 $[(4 k+1) \pi,(4 k+3) \pi](k \geqslant 1)$ 处在 $\left(\cos \frac{w}{2}\right)^{2 n+\theta}$ 的割线处, 函数 $f(w)$ 在区间 $[(4 k+1) \pi$, $(4 k+3) \pi](k \geqslant 1)$ 上不解析, 但是, 由于 $f(w)$ 的对称性, 割线上下两岸的积分最后相互抵消了.

因此,

$$
2 \int_{-\infty-\mathrm{i} A}^{\infty-\mathrm{i} A} f(w) d w=\sum_{k \in \mathbb{Z}} \int_{C_{4 k+1}} f(w) d w+\sum_{k \in \mathbb{Z}} \int_{C_{4 k-1}} f(w) d w
$$




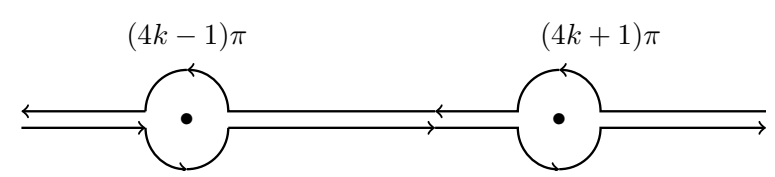

图 1 Fourier 积分围道变形

这里 $C_{4 k \pm 1}$ 是圆心在 $(4 k \pm 1) \pi$ 、半径为 1 的圆周. 于是, 若记单位圆周为 $C$, 则

$$
\begin{aligned}
2 \int_{-\infty-\mathrm{i} A}^{\infty-\mathrm{i} A} f(w) d w & =\int_{C}\left[\sum_{k \in \mathbb{Z}} \mathrm{e}^{-\frac{(4 k \pi+\pi+z)^{2}}{2 t}} \frac{\mathrm{i} \sin \left(\frac{z+\pi}{2}\right)}{\cos ^{2 n+\theta}\left(\frac{z+\pi}{2}\right)}-\sum_{k \in \mathbb{Z}} \mathrm{e}^{-\frac{(4 k \pi+\pi-z)^{2}}{2 t}} \frac{\mathrm{i} \sin \left(\frac{\pi-z}{2}\right)}{\cos ^{2 n+\theta}\left(\frac{\pi-z}{2}\right)}\right] d z \\
& =\mathrm{i} \int_{C}\left[K_{t}(z) \phi_{t}(z)-K_{t}(-z) \phi_{t}(-z)\right] d z .
\end{aligned}
$$

由此, 定理得证.

\section{1 定理 3.1 的证明}

定理 3.1 的证明 利用定理 3.2 中的积分表示进行渐近分析. 由定理 3.2 可知,

$$
\mathrm{P}\left(D_{t}=n\right)=\left(\begin{array}{c}
2 n-1+\theta \\
n
\end{array}\right) \frac{c_{n}^{\theta}(t)}{2^{2 n-1+\theta}} .
$$

由 Stirling 公式可知,

$$
\left(\begin{array}{c}
2 n-1+\theta \\
n
\end{array}\right) \frac{1}{2^{2 n-1+\theta}} \sim \frac{1}{\sqrt{2 \pi}} \sqrt{\frac{2}{n}}
$$

接下来主要对积分

$$
c_{n}^{\theta}(t)=\mathrm{e}^{\frac{(1-\theta)^{2} t}{8}} \frac{\mathrm{i}}{2 \sqrt{2 \pi t}} \int_{C}\left[K_{t}(z) \phi_{t}(z)-K_{t}(-z) \phi_{t}(-z)\right] d z
$$

进行渐近分析. 首先, 做围道变形; 其次, 对被积函数做渐近展开.

第 1 步 围道变形考虑到函数 $K_{t}(z) \phi_{t}(z)-K_{t}(-z) \phi_{t}(-z)$ 是奇函数. 那么,

$$
\begin{aligned}
\int_{C} & {\left[K_{t}(z) \phi_{t}(z)-K_{t}(-z) \phi_{t}(-z)\right] d z } \\
& =\int_{C_{+}}\left[K_{t}(z) \phi_{t}(z)-K_{t}(-z) \phi_{t}(-z)\right] d z+\int_{C_{-}}\left[K_{t}(z) \phi_{t}(z)-K_{t}(-z) \phi_{t}(-z)\right] d z \\
& =2 \int_{C_{+}}\left[K_{t}(z) \phi_{t}(z)-K_{t}(-z) \phi_{t}(-z)\right] d z,
\end{aligned}
$$

这里 $C_{+}$是上半圆周, 其方向为逆时针方向. 因此,

$$
c_{n}^{\theta}(t)=\mathrm{e}^{\frac{(1-\theta)^{2} t}{8}} \frac{\mathrm{i}}{\sqrt{2 \pi t}} \int_{C+}\left[K_{t}(z) \phi_{t}(z)-K_{t}(-z) \phi_{t}(-z)\right] d z .
$$

考虑如下两个围道 (见图 2):

$$
L_{1}: z(y)= \begin{cases}-\pi+y+\mathrm{i} y, & 0 \leqslant y \leqslant 1+\pi \\ \pi+\mathrm{i} y, & 1+\pi \leqslant y \leqslant 0\end{cases}
$$




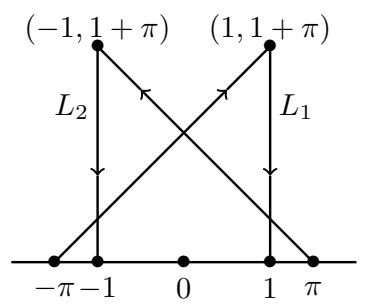

图 2 最速下降围道

$$
L_{2}: z(y)= \begin{cases}1-y+\mathrm{i} y, & 0 \leqslant y \leqslant 1+\pi, \\ -1+\mathrm{i} y, & 1+\pi \leqslant y \leqslant 0 .\end{cases}
$$

首先将 $\int_{C_{+}} K_{t}(z) \phi_{t}(z) d z$ 的围道向右扩大到 $L_{1}$, 然后将 $\int_{C_{+}} K_{t}(-z) \phi_{t}(-z) d z$ 的积分轨道向左扩 张到 $L_{2}$. 由于上述变形都在各自的解析区域进行, 故积分不变. 注意到 $L_{1}$ 的取向与 $C_{+}$的取向相反, 以及 $L_{1}$ 和 $L_{2}$ 的对称性, 可知

$$
\int_{C+}\left[K_{t}(z) \phi_{t}(z)-K_{t}(-z) \phi_{t}(-z)\right] d z=-\int_{L_{1}} K_{t}(z) \phi_{t}(z) d z+\int_{L_{1}} K_{t}(\bar{z}) \phi_{t}(\bar{z}) d \bar{z} .
$$

$K_{t}(z)$ 可以重新表述为

$$
K_{t}(z)=\exp \left\{\frac{1}{t} \psi_{t}\left(\frac{z+\pi}{2}\right)\right\} \sin \frac{z+\pi}{2}
$$

这里

$$
\psi_{t}(z)=-2 z^{2}-(2 n+\theta) t \log \cos (z) .
$$

类似地,

$$
K_{t}(\bar{z})=\exp \left\{\frac{1}{t} \psi_{t}\left(\frac{\bar{z}+\pi}{2}\right)\right\} \sin \frac{\bar{z}+\pi}{2} .
$$

可以证明 $\psi_{t}\left(\frac{\pi+z}{2}\right)$ 和 $\psi_{t}\left(\frac{\pi+\bar{z}}{2}\right)$ 分别是 $K_{t}(z)$ 和 $K_{t}(\bar{z})$ 的主要部分, 因为被积函数的模

$$
\left|\exp \left\{\frac{1}{t} \psi_{t}\left(\frac{z+\pi}{2}\right)\right\}\right|=\left|\exp \left\{\frac{1}{t} \psi_{t}\left(\frac{\pi+\bar{z}}{2}\right)\right\}\right|=\exp \left\{\frac{1}{t} \operatorname{Re}\left[\psi_{t}\left(\frac{z+\pi}{2}\right)\right]\right\}
$$

在 $-\pi$ 处取到最大值, 然后沿 $L_{1}$ 逐渐递减. 称 $L_{1}$ 为最速下降轨道. 事实上, 由于 $\psi_{t}^{\prime}(z)=-4 z+(2 n$ $+\theta) t \tan (z)$, 故 $\psi_{t}^{\prime}(0)=0$, 所以, $z=-\pi$ 是 $\psi_{t}\left(\frac{z+\pi}{2}\right)$ 的临界点. 另外, 当 $z(y)=-\pi+y+\mathrm{i} y$, 则

$$
a(y):=\operatorname{Re}\left[\psi_{t}\left(\frac{z(y)+\pi}{2}\right)\right]=-\frac{(2 n+\theta) t}{2} \log \frac{\cosh y+\cos y}{2} .
$$

易证 $a^{\prime}(y)=-(2 n+\theta) t \frac{\sinh y-\sin y}{\cosh y+\cos y} \leqslant 0, \forall 0 \leqslant y \leqslant 1+\pi$. 于是, $a(y)$ 关于 $y$ 单调递减. 另外, 可以证明 在 $L_{1}$ 的坚直部分, $a(y)$ 的最大值在端点 $1+\mathrm{i}(1+\pi)$ 处取到, 故小于临界点处的取值. 因此, $L_{1}$ 是最 速下降轨道.

第 2 步 渐近展开.

首先将 $\phi_{t}(z)$ 和 $\phi_{t}(\bar{z})$ 用常值函数 1 来逼近, 可以证明其误差是指数递降的. 
引理 3.1 定义 $R_{1}=\int_{L_{1}} K_{t}(z)\left(\phi_{t}(z)-1\right) d z$, 则存在正常数 $M_{1}$ 使得

$$
\left|R_{1}\right| \leqslant \frac{M_{1} \mathrm{e}^{-\frac{4 \pi(\pi-1)}{t}}}{1-\mathrm{e}^{-\frac{4 \pi(\pi-1)}{t}}} .
$$

其次, 将 $L_{1}$ 上的积分分成两部分. 记 $L_{1, t}$ 为靠近 $-\pi$ 的一小段直线, 其参数方程为

$$
-\pi+2 t^{\frac{1}{4}} \mathrm{e}^{\mathrm{i} \pi / 4} \sqrt{y}, \quad y \in\left(0, t^{-2 \epsilon}\right), \quad \epsilon<\frac{1}{4} .
$$

记 $L_{1, t}^{c}$ 为剩下的一段 $L_{1}-L_{1, t}$. 可以证明 $L_{1}$ 上的积分的主要贡献来自 $L_{1, t}$, 并且在 $L_{1, t}^{c}$ 上的积分 $R_{2}=\int_{L_{1, t}^{c}} K_{t}(z) d z$ 是指数递降的.

引理 3.2 存在 $M_{2}, M_{3}, \delta_{1}>0$, 使得 $\forall 0<t<\delta_{1}$,

$$
\left|R_{2}\right|=\left|\int_{L_{1, t}^{c}} K_{t}(z) d z\right|=\left|\int_{L_{1, t}^{c}} K_{t}(\bar{z}) d \bar{z}\right| \leqslant M_{2} \exp \left\{-M_{3} \frac{1}{t^{4 \epsilon}}\right\},
$$

这里 $M_{2}$ 和 $M_{3}$ 是不依赖于 $t$ 的两个参数.

最后, 将对 $L_{1, t}$ 上的积分做渐近展开. 由于

$$
\psi_{t}(z)=\left[-2+\frac{(2 n+\theta) t}{2}\right] z^{2}+(2 n+\theta) t \sum_{k=2}^{\infty}(-1)^{k-1} \frac{2^{2 k}\left(2^{2 k}-1\right) B_{2 k}}{2 k(2 k) !} z^{2 k},
$$

这里 $B_{2 k}$ 是 Bernoulli 数 (参见文献 [14, 第 75 页, (4.3.72)]), 于是, 对于任意正整数 $m$ 和 $z \in L_{1, t}$, 有

$$
\begin{aligned}
\exp \left\{\frac{1}{t} \psi_{t}\left(\frac{z+\pi}{2}\right)\right\}= & \exp \left\{\left[\frac{(2 n+\theta) t-4}{2 t}\right]\left(\frac{z+\pi}{2}\right)^{2}+\frac{(2 n+\theta)}{12}\left(\frac{z+\pi}{2}\right)^{4}\right\} \\
& \times \exp \left\{-g(w)+\Delta_{0}\right\}
\end{aligned}
$$

这里

$$
g(w)=(2 n+\theta) \sum_{k=3}^{m}(-1)^{k} \frac{2^{2 k}\left(2^{2 k}-1\right) B_{2 k}}{2 k(2 k) !} w^{k}, \quad w=\left(\frac{z+\pi}{2}\right)^{2} .
$$

若记 $a_{k}=\frac{2^{2 k+4}\left(2^{2 k+4}-1\right) B_{2 k+4} k !}{(2 k+4)(2 k+4) !}$, 则

$$
g(w)=\sum_{k=1}^{m-2}(-1)^{k} \frac{a_{k}(2 n+\theta)\left(\frac{z+\pi}{2}\right)^{4}}{k !} w^{k}
$$

并且存在 $M_{4}, \delta_{2}>0$, 使得当 $0<t<\delta_{2}$ 时,

$$
|g(w)|+\left|\Delta_{0}\right| \leqslant \max \left\{M_{4}(2 n+\theta)\left|\frac{z+\pi}{2}\right|^{6}, \frac{(2 n+\theta)\left|\frac{z+\pi}{2}\right|^{4}}{36}\right\} .
$$

当把 $\mathrm{e}^{-g(w)} \frac{\sin \left(\frac{z+\pi}{2}\right)}{\frac{z+\pi}{2}}$ 在 $z=-\pi$ 处展开后, 就得到 $K_{t}(z)$ 的渐近展开式.

引理 3.3 定义 $c_{0}(z)=1$,

$$
c_{k}(z)=(-1)^{k} \sum_{l=0}^{k} \frac{1}{(2(k-l)+1) !}\left\{\sum_{\mathcal{S}_{l}} \prod_{i=1}^{l} \frac{\left(a_{i} / i !\right)^{m_{i}}}{m_{i} !}\left[-(2 n+\theta)\left(\frac{z+\pi}{2}\right)^{4}\right]^{\sum_{i=1}^{l} m_{i}}\right\}, \quad k \geqslant 1,
$$


那么, $K_{t}(z)$ 有展开式

$$
K_{t}(z)=\exp \left\{\left[\frac{(2 n+\theta) t-4}{2 t}\right]\left(\frac{z+\pi}{2}\right)^{2}+\frac{2 n+\theta}{12}\left(\frac{z+\pi}{2}\right)^{4}\right\} \sum_{k=0}^{m-2} c_{k}(z)\left(\frac{z+\pi}{2}\right)^{2 k+1}+\Delta(z) .
$$

若记 $R_{3}=\int_{L_{1, t}} \Delta(z) d z$, 则存在 $M_{5}, \delta_{4}>0$, 当 $0<t<\delta_{4}$ 时,

$$
\left|R_{3}\right|=\left|\int_{L_{1, t}} \Delta(\bar{z}) d \bar{z}\right| \leqslant M_{5} t^{m / 2}
$$

由上述引理可知, 积分 $\int_{L_{1, t}} K_{t}(z) d z$ 和 $\int_{L_{1, t}} K_{t}(\bar{z}) d \bar{z}$ 可以分别由

$$
\left.A=\int_{L_{1, t}} \exp \left\{\left[\frac{(2 n+\theta) t-4}{2 t}\right]\left(\frac{z+\pi}{2}\right)^{2}+\frac{2 n+\theta}{12}\left(\frac{z+\pi}{2}\right)^{4}\right\} \sum_{k=0}^{m-2} c_{k}(z)\left(\frac{z+\pi}{2}\right)^{2 k+1}\right] d z
$$

和

$$
\left.B=\int_{L_{1, t}} \exp \left\{\left[\frac{(2 n+\theta) t-4}{2 t}\right]\left(\frac{\bar{z}+\pi}{2}\right)^{2}+\frac{2 n+\theta}{12}\left(\frac{\bar{z}+\pi}{2}\right)^{4}\right\} \sum_{k=0}^{m-2} c_{k}(\bar{z})\left(\frac{\bar{z}+\pi}{2}\right)^{2 k+1}\right] d \bar{z}
$$

逼近. 考虑到 $L_{1, t}$ 的参数表示 $z(y)=-\pi+2 t^{\frac{1}{4}} \sqrt{y} \mathrm{e}^{\mathrm{i} \pi / 4}, 0 \leqslant y \leqslant \frac{1}{t^{2 \epsilon}}$, 可知

$$
\begin{aligned}
& A=t^{\frac{1}{2}} \mathrm{i} \int_{0}^{\frac{1}{t^{2 \epsilon}}} \exp \left\{\left[\frac{(2 n+\theta) t-4}{2 \sqrt{t}}\right] y \mathrm{i}-\frac{(2 n+\theta) t}{12} y^{2}\right\} \sum_{k=0}^{m-2} c_{k}(z(y))(\sqrt{t} y \mathrm{i})^{k} d y, \\
& B=-t^{\frac{1}{2}} \mathrm{i} \int_{0}^{\frac{1}{t^{2 \epsilon}}} \exp \left\{-\left[\frac{(2 n+\theta) t-4}{2 \sqrt{t}}\right] y \mathrm{i}-\frac{(2 n+\theta) t}{12} y^{2}\right\} \sum_{k=0}^{m-2} c_{k}(\overline{z(y)})(-\sqrt{t} y \mathrm{i})^{k} d y .
\end{aligned}
$$

对 $B$ 进行变量代换可知,

$$
\begin{aligned}
A-B= & t^{\frac{1}{2}} \mathrm{i} \int_{-\frac{1}{t^{2 \epsilon}}}^{\frac{1}{t^{2 \epsilon}}} \exp \left\{\left[\frac{(2 n+\theta) t-4}{2 \sqrt{t}}\right] y \mathrm{i}-\frac{(2 n+\theta) t}{12} y^{2}\right\} \sum_{k=0}^{m-2} c_{k}(z(y))(-\sqrt{t} y \mathrm{i})^{k} d y \\
= & t^{\frac{1}{2}} \mathrm{i} \int_{-\infty}^{\infty} \exp \left\{\left[\frac{(2 n+\theta) t-4}{2 \sqrt{t}}\right] y \mathrm{i}-\frac{(2 n+\theta) t}{12} y^{2}\right\} \sum_{k=0}^{m-2} c_{k}(z(y))(-\sqrt{t} y \mathrm{i})^{k} d y \\
& +R_{4},
\end{aligned}
$$

并且 $\left|R_{4}\right| \leqslant M_{6} \mathrm{e}^{-M_{7} / t^{4 \epsilon}}, M_{6}, M_{7}>0$. 于是, 由于 $\sigma^{2}=\frac{6}{(2 n+\theta) t}, v=\frac{(2 n+\theta) t-4}{2 \sqrt{t}}$, 因此,

$$
\begin{aligned}
\int_{C+} & {\left[K_{t}(z) \phi_{t}(z)-K_{t}(-z) \phi_{t}(-z)\right] d z } \\
= & -\int_{L_{1}} K_{t}(z) \phi_{t}(z) d z+\int_{L_{1}} K_{t}(\bar{z}) \phi_{t}(\bar{z}) d \bar{z} \\
= & -\int_{L_{1}} K_{t}(z) d z+\int_{L_{1}} K_{t}(\bar{z}) d \bar{z}+R_{1} \\
= & -\int_{L_{1, t}} K_{t}(z) d z+\int_{L_{1, t}} K_{t}(\bar{z}) d \bar{z}+R_{1}+R_{2} \\
= & -A+B+R_{1}+R_{2}+R_{3}
\end{aligned}
$$




$$
\begin{aligned}
= & -\sqrt{t} \mathrm{i} \int_{-\infty}^{\infty} \mathrm{e}^{v y \mathrm{i}-\frac{\sigma^{2} y^{2}}{2}} \sum_{k=0}^{m-2} c_{k}(z(y))(-\sqrt{t} y \mathrm{i})^{k} d y+R_{1}+R_{2}+R_{3}+R_{4} \\
= & -\sqrt{t} \mathrm{i} \sum_{k=0}^{m-2}(\sqrt{t})^{k} \sum_{l=0}^{k} \frac{1}{(2(k-l)+1) !}\left\{\sum_{\mathcal{S}_{l}} \prod_{i=1}^{l} \frac{\left[a_{i} / i !\right]^{m_{i}}}{m_{i} !}\left(-6 \sigma^{2}\right)^{\sum_{i=1}^{l} m_{i}}\right. \\
& \left.\times \int_{-\infty}^{\infty} \mathrm{e}^{v y \mathrm{i}-\frac{\sigma^{2} y^{2}}{2}}(y \mathrm{i})^{k+2 \sum_{i=1}^{l} m_{i}} d y\right\}+R_{1}+R_{2}+R_{3}+R_{4} .
\end{aligned}
$$

由 Fourier 变换可知,

$$
\int_{-\infty}^{\infty} \mathrm{e}^{v y \mathrm{i}-\frac{\sigma^{2} y^{2}}{2}} d y=\frac{\sqrt{2 \pi}}{\sigma} \mathrm{e}^{-\frac{v^{2}}{2 \sigma^{2}}}
$$

若对等式两边关于 $v$ 求 $k+2 \sum_{i=1}^{l} m_{i}$ 次导数, 则得到

$$
\int_{-\infty}^{\infty} \mathrm{e}^{v y \mathrm{i}-\frac{\sigma^{2} y^{2}}{2}}(y \mathrm{i})^{k+2 \sum_{i=1}^{l} m_{i}} d y=\frac{\sqrt{2 \pi}}{\sigma} \frac{1}{\sigma^{k+2 \sum_{i=1}^{l} m_{i}}}(-1)^{k+2 \sum_{i=1}^{l} m_{i}} H_{k+2 \sum_{i=1}^{l} m_{i}}\left(\frac{v}{\sigma}\right) \mathrm{e}^{-\frac{v^{2}}{2 \sigma^{2}}},
$$

所以,

$$
\begin{aligned}
& \int_{C+} {\left[K_{t}(z) \phi_{t}(z)-K_{t}(-z) \phi_{t}(-z)\right] d z } \\
&=-\sqrt{t} \mathrm{i} \frac{\sqrt{2 \pi}}{\sigma} \mathrm{e}^{-\frac{v^{2}}{2 \sigma^{2}}} \sum_{k=0}^{m-2}\left(-\frac{\sqrt{t}}{\sigma}\right)^{k} \sum_{l=0}^{k} \frac{1}{(2(k-l)+1) !}\left\{\sum_{\mathcal{S}_{l}} \prod_{i=1}^{l} \frac{\left[-6 a_{i} / i !\right]^{m_{i}}}{m_{i} !} H_{k+2 \sum_{i=1}^{l} m_{i}}\left(\frac{v}{\sigma}\right)\right\} \\
& \quad+R_{1}+R_{2}+R_{3}+R_{4} .
\end{aligned}
$$

再由等式 (3.1), 得到

$$
\mathrm{P}\left(D_{t}=n\right)=\sum_{k=0}^{m-2}(-1)^{k} t^{(k+1) / 2} d_{k}(v) \frac{1}{\sqrt{2 \pi} \sigma} \mathrm{e}^{-\frac{v^{2}}{2 \sigma^{2}}}+R_{5},
$$

这里 $\left|R_{5}\right| \leqslant M_{9} t^{(m+1) / 2}$,

$$
d_{k}(v)=\mathrm{e}^{\frac{(1-\theta)^{2} t}{8}}\left(\begin{array}{c}
2 n-1+\theta \\
n
\end{array}\right) \frac{\sqrt{2 \pi}}{\sqrt{t} \sigma^{k} 2^{2 n-1+\theta}} \sum_{l=0}^{k} \frac{1}{(2(k-l)+1) !}\left\{\sum_{\mathcal{S}_{l}} \frac{\left[-6 a_{i} / i !\right]^{m_{i}}}{m_{i} !} H_{k+2 \sum_{i=1}^{k} m_{i}}\left(\frac{v}{\sigma}\right)\right\} .
$$

注意, 由于 $n \sim \frac{2}{t}$, 当 $t \rightarrow 0$ 时, 有

$$
\left(\begin{array}{c}
2 n-1+\theta \\
n
\end{array}\right) \frac{\sqrt{2 \pi}}{\sqrt{t} 2^{2 n-1+\theta}} \sim 1 .
$$

于是得到 $\mathrm{P}\left(D_{t}=n\right)$ 的渐近展开式

$$
\mathrm{P}\left(D_{t}=n\right) \sim \sum_{k=0}^{\infty}(-1)^{k}\left[d_{k}(v) \frac{1}{\sqrt{2 \pi} \sigma} \mathrm{e}^{-\frac{v^{2}}{2 \sigma^{2}}}\right] t^{(k+1) / 2} .
$$

证毕. 


\section{4 引理的证明}

引理 3.1 的证明 注意到 $R_{1}=\int_{L_{1}} K_{t}(z)\left(\phi_{t}(z)-1\right) d z$, 所以, 存在正常数 $M_{1}$, 使得

$$
\begin{aligned}
\left|R_{1}\right| & \leqslant M_{1} \exp \left\{\frac{1}{t} \sup _{z \in L_{1}} \operatorname{Re}\left(\psi_{t}\left(\frac{z+\pi}{2}\right)\right)\right\} \sup _{z \in L_{1}}\left|\phi_{t}(z)-1\right| \sup _{z \in L_{1}}\left|\sin \frac{\pi+z}{2}\right| \\
& \leqslant M_{1} \sup _{z \in L_{1}}\left|\phi_{t}(z)-1\right| \leqslant M_{1} \sum_{k=1}^{\infty} \mathrm{e}^{-\frac{8 k^{2} \pi^{2}}{t}} \sup _{z \in L_{1}}\left|\cosh \frac{4 k \pi(z+\pi)}{t}\right| \\
& \leqslant M_{1} \sum_{k=1}^{\infty} \mathrm{e}^{-\frac{8 k^{2} \pi^{2}}{t}} \mathrm{e}^{\frac{4 k \pi(1+\pi)}{t}} \leqslant M_{1} \sum_{k=1}^{\infty} \mathrm{e}^{-\frac{4 k \pi(\pi-1)}{t}} \\
& \leqslant \frac{M_{1} \mathrm{e}^{-\frac{4 \pi(\pi-1)}{t}}}{1-\mathrm{e}^{-\frac{4 \pi(\pi-1)}{t}}} .
\end{aligned}
$$

证毕.

引理 3.2 的证明 存在 $M_{2}>0$, 使得

$$
\left|R_{2}\right|=\left|\int_{L_{1, t}^{c}} K_{t}(z) d z\right|=\left|\int_{L_{1, t}^{c}} K_{t}(\bar{z}) d \bar{z}\right| \leqslant M_{2} \exp \left\{\frac{1}{t} \sup _{z \in L_{1, t}^{c}} \operatorname{Re}\left[\psi_{t}\left(\frac{z+\pi}{2}\right)\right]\right\} .
$$

再由 $\operatorname{Re}\left[\psi_{t}\left(\frac{z+\pi}{2}\right)\right]$ 在 $L_{1}$ 上的单调性可知,

$$
\begin{aligned}
\frac{1}{t} \sup _{z \in L_{1, t}^{c}} \operatorname{Re}\left[\psi_{t}\left(\frac{z+\pi}{2}\right)\right] & =\frac{1}{t} \operatorname{Re}\left[\psi_{t}\left(t^{\frac{1}{4}-\epsilon} \mathrm{e}^{\mathrm{i} \frac{\pi}{4}}\right)\right] \\
& =-\frac{2 n+\theta}{2} \log \frac{\cosh \left(\sqrt{2} t^{\frac{1}{4}}-\epsilon\right)+\cos \left(\sqrt{2} t^{\frac{1}{4}-\epsilon}\right)}{2} \\
& \sim-\frac{(2 n+\theta) t}{12} \frac{1}{t^{4 \epsilon}} .
\end{aligned}
$$

所以, 一定存在 $M_{3}, \delta_{1}>0$, 使得对于 $0<t<\delta_{1}$, 有

$$
\frac{1}{t} \sup _{z \in L_{1, t}^{c}} \operatorname{Re}\left[\psi_{t}\left(\frac{z+\pi}{2}\right)\right] \leqslant-M_{3} \frac{1}{t^{4 \epsilon}} .
$$

于是, 引理得证.

引理 3.3 的证明 首先展开 $f(w)=\mathrm{e}^{-g(w)}$, 于是,

$$
f(w)=\sum_{p=0}^{m} \frac{(-1)^{p}}{p !} g^{p}(w)+\Delta_{1} .
$$

由不等式 $\left|\mathrm{e}^{z}-\sum_{p=0}^{m} \frac{z^{p}}{p !}\right| \leqslant \frac{|z|^{m}}{m !} \mathrm{e}^{|z|}$ 和 (3.3) 可知, 存在 $\delta>0$, 使得当 $0<t<\delta$ 时,

$$
\left|\Delta_{1}\right| \leqslant \frac{|g(w)|^{m}}{m !} \mathrm{e}^{|g(w)|} \leqslant M\left[(2 n+\theta)\left|\frac{\pi+z}{2}\right|^{6}\right]^{m} \mathrm{e}^{\frac{(2 n+\theta)}{36}\left|\frac{\pi+z}{2}\right|^{4}} .
$$

然后

$$
[g(w)]^{p}=\sum_{\sum_{i=1}^{m-2} u_{i}=p} \frac{p !}{u_{1} ! \cdots u_{m-2} !} \prod_{i=1}^{m-2}\left[(-1)^{i} \frac{(2 n+\theta)\left(\frac{z+\pi}{2}\right)^{4} a_{i}}{i !}\right]^{u_{i}} w^{\sum_{i=1}^{m-2} i u_{i}}
$$


于是, 按照 $w^{\sum_{i=1}^{m-2} i u_{i}}$ 的幕次将 $\sum_{p=0}^{m} \frac{(-1)^{p}}{p !} g^{p}(w)$ 分成 $\Delta_{2}+\Delta_{3}$, 这里

$$
\begin{aligned}
& \Delta_{2}=\sum_{p=0}^{m-2} \frac{(-1)^{p}}{p !} \sum_{\substack{\sum_{i=1}^{m-2} u_{i}=p \\
\sum_{i=1}^{m-2} i u_{i} \leqslant m-2}} \frac{p !}{u_{1} ! \cdots u_{m-2} !} \prod_{i=1}^{m-2}\left[(-1)^{i} \frac{(2 n+\theta)\left(\frac{z+\pi}{2}\right)^{4} a_{i}}{i !}\right]^{u_{i}} w^{\sum_{i=1}^{m-2} i u_{i}}, \\
& \Delta_{3}=\sum_{p=2}^{m-2} \frac{(-1)^{p}}{p !} \sum_{\substack{\sum_{i=1}^{m-2} u_{i}=p \\
\sum_{i=1}^{m-1} i u_{i}>m-2}} \frac{p !}{u_{1} ! \cdots u_{m-2} !} \prod_{i=1}^{m-2}\left[(-1)^{i} \frac{(2 n+\theta)\left(\frac{z+\pi}{2}\right)^{4} a_{i}}{i !}\right]^{u_{i}} w^{\sum_{i=1}^{m-2} i u_{i}} .
\end{aligned}
$$

将 $\Delta_{2}$ 合并同类项, 便得到 $f(w)$ 的展开式 $f(w)=\sum_{k=0}^{m-2} \frac{b_{k}}{k !} w^{k}+\Delta_{4}$, 稍后讨论系数 $b_{k}$ 的表达式. 要 知道, $\Delta_{3}$ 应放在误差里, 所以, $\Delta_{4}=\Delta_{1}+\Delta_{3}$. 注意到 $w=\left(\frac{z+\pi}{2}\right)^{2}$, 于是,

$$
\begin{aligned}
& \left|\Delta_{3}\right| \leqslant M|w|^{m-1} \sum_{p=2}^{m-2}\left[(2 n+\theta)\left|\frac{z+\pi}{2}\right|^{4}\right]^{p} \\
& \left|\Delta_{1}\right| \leqslant M|w|^{m}\left[(2 n+\theta)\left|\frac{z+\pi}{2}\right|^{4}\right]^{m} \mathrm{e}^{\frac{(2 n+\theta)}{36}\left|\frac{\pi+z}{2}\right|^{4}},
\end{aligned}
$$

故

$$
\left|\Delta_{4}\right| \leqslant M|w|^{m-1} \sum_{p=2}^{m}\left[(2 n+\theta)\left|\frac{z+\pi}{2}\right|^{4}\right]^{p} \mathrm{e}^{\frac{(2 n+\theta)}{36}\left|\frac{\pi+z}{2}\right|^{4}} .
$$

事实上, 由 Taylor 展开式系数的唯一性可知, $b_{k}=f^{(k)}(0)$. 若记 $h(z)=\mathrm{e}^{-z}$, 则 $f(w)=h(g(w))$, 然后 由 Faá di Bruno 公式 (参见文献 [14, 第 823 页])

$$
f^{(k)}(0)=\sum_{\mathcal{S}_{k}} \frac{k !}{m_{1} ! \cdots m_{k} ! 1 !^{m_{1}} \cdots k !^{m_{k}}} h^{\left(\sum_{i=1}^{k} m_{i}\right)}(g(0)) \prod_{i=1}^{k}\left[g^{(i)}(0)\right]^{m_{i}}
$$

可知,

$$
\begin{aligned}
& b_{0}=1, \\
& b_{k}=k !(-1)^{k} \sum_{\mathcal{S}_{k}} \prod_{i=1}^{k} \frac{\left[a_{i} / i !\right]^{m_{i}}}{m_{i} !}\left[-(2 n+\theta)\left(\frac{z+\pi}{2}\right)^{4}\right]^{\sum_{i=1}^{k} m_{i}}, \quad k \geqslant 1 .
\end{aligned}
$$

最后, 将 $\sin \left(\frac{z+\pi}{2}\right) /\left(\frac{z+\pi}{2}\right)$ 在 $z=-\pi$ 处展开可得

$$
\frac{\sin \left(\frac{z+\pi}{2}\right)}{\frac{z+\pi}{2}}=\sum_{k=0}^{m-2}(-1)^{k} \frac{w^{k}}{(2 k+1) !}+\Delta_{5},
$$

这里 $\left|\Delta_{5}\right| \leqslant M|w|^{m-1}$. 再由幂级数的乘积公式可知, $f(w) \sin \left(\frac{z+\pi}{2}\right) /\left(\frac{z+\pi}{2}\right)$ 具有如下展开式:

$$
\frac{f(w) \sin \left(\frac{z+\pi}{2}\right)}{\frac{z+\pi}{2}}=\sum_{k=0}^{m-2} c_{k} w^{k}+\Delta_{6}
$$

这里

$$
c_{k}=(-1)^{k} \sum_{l=0}^{k} \frac{1}{(2(k-l)+1) !}\left\{\sum_{\mathcal{S}_{l}} \prod_{i=1}^{l} \frac{\left(a_{i} / i !\right)^{m_{i}}}{m_{i} !}\left[-(2 n+\theta)\left(\frac{z+\pi}{2}\right)^{4}\right]^{\sum_{i=1}^{l} m_{i}}\right\},
$$




$$
\Delta_{6}=\left[\sum_{k=0}^{m-2} \frac{b_{k}}{k !} w^{k}\right] \Delta_{5}+\frac{\Delta_{4} \sin \left(\frac{z+\pi}{2}\right)}{\frac{z+\pi}{2}} .
$$

注意到

$$
\sum_{k=0}^{m-2} \frac{b_{k}}{k !} w^{k}=\Delta_{2}=\sum_{p=0}^{m-2} \frac{(-1)^{p}}{p !}[g(w)]^{p}-\Delta_{3},
$$

所以, 由不等式

$$
\left|\sum_{k=0}^{m-2} \frac{z^{k}}{k !}\right| \leqslant\left(1+\frac{|z|^{m-1}}{(m-1) !}\right) \mathrm{e}^{|z|}
$$

可知,

$$
\begin{aligned}
\left|\sum_{k=0}^{m-2} \frac{b_{k}}{k !} w^{k}\right| \leqslant & M\left(1+|g(w)|^{m-1}\right) \mathrm{e}^{|g(w)|}+\left|\Delta_{3}\right| \\
\leqslant & M\left[1+(2 n+\theta)^{m-1}\left|\frac{z+\pi}{2}\right|^{4(m-1)}\right] \mathrm{e}^{\left.\frac{(2 n+\theta) \mid \frac{\pi+z}{2}}{36}\right|^{4}} \\
& +M|w|^{m-1} \sum_{p=2}^{m-2}\left[(2 n+\theta)\left|\frac{z+\pi}{2}\right|^{4}\right]^{p} \\
\leqslant & M\left(\sum_{s=0}^{m}\left[(2 n+\theta)\left|\frac{z+\pi}{2}\right|^{4}\right]^{s}\right) \mathrm{e}^{\frac{(2 n+\theta)\left|\frac{\pi+z}{2}\right|^{4}}{36}}
\end{aligned}
$$

于是,

$$
\left|\Delta_{6}\right| \leqslant M\left(\sum_{s=0}^{m}\left[(2 n+\theta)\left|\frac{z+\pi}{2}\right|^{4}\right]^{s}\right) \mathrm{e}^{\frac{(2 n+\theta)\left|\frac{\pi+z}{2}\right|^{4}}{36}}|w|^{m-1} .
$$

最后, 由于

$$
\begin{aligned}
K_{t}(z)= & \exp \left\{\frac{1}{t} \psi_{t}\left(\frac{z+\pi}{2}\right)\right\} \sin \frac{z+\pi}{2} \\
= & \exp \left\{\left[\frac{(2 n+\theta) t-4}{2 t}\right]\left(\frac{z+\pi}{2}\right)^{2}+\frac{2 n+\theta}{12}\left(\frac{z+\pi}{2}\right)^{4}\right\} \\
& \times \frac{z+\pi}{2} \exp \{-g(w)\} \frac{\sin \frac{z+\pi}{2}}{\frac{z+\pi}{2}} \exp \left\{\Delta_{0}\right\} \\
= & \frac{z+\pi}{2} \exp \left\{\left[\frac{(2 n+\theta) t-4}{2 t}\right]\left(\frac{z+\pi}{2}\right)^{2}+\frac{2 n+\theta}{12}\left(\frac{z+\pi}{2}\right)^{4}\right\} \sum_{k=0}^{m-2} c_{k} w^{k}+\Delta_{7},
\end{aligned}
$$

这里

$$
\Delta_{7}=\frac{z+\pi}{2} \exp \left\{\left[\frac{(2 n+\theta) t-4}{2 t}\right]\left(\frac{z+\pi}{2}\right)^{2}+\frac{2 n+\theta}{12}\left(\frac{z+\pi}{2}\right)^{4}\right\} R_{4} \exp \left\{\Delta_{0}\right\}
$$

所以,

$$
\begin{aligned}
& \left|\int_{L_{1, t}}\left[K_{t}(z)-\exp \left\{\left[\frac{(2 n+\theta) t-4}{2 t}\right]\left(\frac{z+\pi}{2}\right)^{2}+\frac{2 n+\theta}{12}\left(\frac{z+\pi}{2}\right)^{4}\right\} \sum_{k=0}^{m-2} c_{k}(z) w^{k}\right] d z\right| \\
& \quad=\left|\int_{L_{1, t}} \exp \left\{\left[\frac{(2 n+\theta) t-4}{2 t}\right]\left(\frac{z+\pi}{2}\right)^{2}+\frac{2 n+\theta}{12}\left(\frac{z+\pi}{2}\right)^{4}\right\} \Delta_{6} \exp \left\{\Delta_{0}\right\} \frac{z+\pi}{2} d z\right|
\end{aligned}
$$




$$
\begin{aligned}
& \leqslant\left|\int_{0}^{\frac{1}{t^{2 \epsilon}}} \mathrm{e}^{-\frac{(2 n+\theta) t}{12} y^{2}} \Delta_{6}(z(y)) \exp \left\{\Delta_{0}(z(y))\right\} t^{1 / 2} \mathrm{e}^{\mathrm{i} \pi / 2} d y\right| \\
& \leqslant M t^{1 / 2}(\sqrt{t})^{m-1}\left|\int_{0}^{\frac{1}{t^{2 \epsilon}}} \mathrm{e}^{-\frac{(2 n+\theta) t}{12} y^{2}} y^{m-1} \mathrm{e}^{\frac{2(2 n+\theta) t}{36}} y^{2} \sum_{s=0}^{m} y^{s} d y\right| \\
& \leqslant M(\sqrt{t})^{m}\left|\int_{0}^{\frac{1}{t^{2 \epsilon}}} \mathrm{e}^{-\frac{(2 n+\theta) t}{36} y^{2}} \sum_{s=0}^{m} y^{s+m-1} d y\right| \\
& \leqslant M D(\sqrt{t})^{m}
\end{aligned}
$$

这里

$$
D=\int_{0}^{\infty} \mathrm{e}^{-\frac{(2 n+\theta) t}{36} y^{2}} \sum_{s=0}^{m} y^{s+m-1} d y<\infty
$$

证毕.

\section{5 结论}

本文得到了 $d_{n}^{\theta}(t)$ 的复积分表示. 作为应用之一, 利用最速下降法得到了局部中心极限定理的渐 近展开式. 除此之外, 该复积分表示应该还有其他的应用, 例如, 利用复积分表示得到精细中偏差和大 偏差.

致谢 作者感谢审稿人的宝贵意见, 本文因此增色不少。

\section{参考文献}

1 Kingman J F C. The coalescent. Stochastic Process Appl, 1982, 13: 235-248

2 Pitman J. Coalescents with multiple collisions. Ann Probab, 1999, 27: 1870-1902

3 Berestycki N. Recent Progress in Coalescent Theory. Ensaios Matemáticos, vol. 16. Rio de Janeiro: Sociedade Brasileira de Matemática, 2009

4 Aldous D J. Deterministic and stochastic models for coalescence (aggregation and coagulation): A review of the mean-field theory for probabilists. Bernoulli, 1999, 5: 3-48

5 Evans S N. Kingman's coalescent as a random metric space. In: Stochastic Models, vol. 26 (Ottawa, ON, 1998). Providence: Amer Math Soc, 2000, 105-114

6 Ethier S N, Griffiths R C. The transition function of a Fleming-Viot process. Ann Probab, 1993, 21: 1571-1590

7 Tavaré S. Line-of-descent and genealogical processes, and their applications in population genetics models. Theoret Popul Biol, 1984, 26: 119-164

8 Zhou Y. Ergodic inequality of a two-parameter infinitely-many-alleles diffusion model. J Appl Probab, 2015, 52: 238-246

9 Depperschmidt A, Pfaffelhuber P, Scheuringer A. Some large deviations in Kingman's coalescent. Electron Commun Probab, 2015, 20: 1-14

10 Griffiths R C. Asymptotic line-of-descent distributions. J Math Biol, 1984, 21: 67-75

11 Limic V, Talarczyk A. Diffusion limits at small times for coalescents with a Kingman component. Electron J Probab, 2015, 20: $1-20$

12 Limic V, Talarczyk A. Second-order asymptotics for the block counting process in a class of regularly varying $\Lambda$ coalescents. Ann Probab, 2015, 43: 1419-1455

13 Griffiths R C. Coalescent lineage distributions. Adv in Appl Probab, 2006, 38: 405-429

14 Abramowitz M, Stegun I A. Handbook of Mathematical Functions with Formulas, Graphs, and Mathematical Tables. New York: Dover, 1964 


\section{Integral representation for Kingman coalescent}

Youzhou Zhou

Abstract Kingman coalescent is the first coalescent model. It has become a benchmark coalescent model recently, and has been widely studied. This article aims at establishing an alternative integral representation for its distribution. We also utilize this integral representation to get the asymptotic expansion of the local central limit theorem at the small time regime.

Keywords Kingman coalescent, integral representation, local central limit theorem $\operatorname{MSC}(2020) \quad 60 \mathrm{~F} 10,60 \mathrm{C05}$

doi: 10.1360/SCM-2019-0575 